\title{
Numerical investigation of toroidal shock wave focusing in a cylindrical chamber
}

\begin{abstract}
In this paper, focusing of a toroidal shock wave propagating from an annular shock tube into a cylindrical chamber was investigated numerically with the dispersion controlled dissipation (DCD) scheme. The first case for an incident Mach number of 1.5 was conducted and compared with experiments for validation. Then, several cases were calculated for higher incident Mach numbers varying from 2.0 to 5.0 , and complicated flow structures were observed. The numerical study was mainly focused on two aspects: focusing process and flow structures. The process, including diffraction, focusing, and reflection, is displayed to reveal the focusing mechanism, and the flow structures at different incident. Mach numbers are used to demonstrate shock reflection styles and focusing characteristics.
\end{abstract}

Keywords DCD scheme - Toroidal shock wave focusing . Spherical double Mach reflection

PACS 47.40.Ki; 47.40.Nm; 52.35.Tc

\section{Introduction}

Shock wave focusing has been an interesting research area for several decades. A small region of high temperature and pressure around a focal point can be generated for various applications. The explosion-like phenomenon will generate blast that can interact with other waves, which may lead to vortex generation, turbulence mixing and so on. Sod [1]

Communicated by K. Takayama

H. Teng $(\bowtie) \cdot$ Z. Jiang

LHD, Institute of Mechanics, Chinese Academy of Sciences,

Beijing 100080, China

E-mail: hhteng@ustc.edu

Z. Han

Department of Modern Mechanics, University of Science and Technology of China, Hefei 230028, China

S. H. R. Hosseini · K. Takayama

Shock Wave Research Center, Institute of Fluid Science,

Tohoku University, Sendai 980-8577, Japan studied cylindrical shock wave focusing numerically first with the one-dimension model. Takayama et al. [2] investigated the stability of cylindrical shock waves travelling in air experimentally. In 1989, Grönig [3] reviewed shock wave focusing and pointed out that the focusing of cylindrical shock waves is one of the research topics worth trying in future. Jiang and Takayama [4] discussed the propagation of the toroidal shock wave discharged from a coaxial annular shock tube and revealed a special shock reflection. Hosseini and Takayama [5] set up a vertical shock tube to visualize the focusing of a toroidal shock wave of a Mach number 1.5. But due to the difficulty arising from generating high quality toroidal shock waves, they did not carry out experiments of higher Mach number cases, which are more interesting in physics and important in practice.

Based on the CFD validation with the experimental results [5], the present work is devoted to numerical simulations of toroidal shock wave focusing in the cylindrical shock tube to explore further shock physics in focusing. In the numerical simulation, the dispersion controlled dissipation (DCD) scheme [6] is used to discretize the NavierStokes equations for calculation, and then several cases are carried out to examine the development of shock wave focusing and wave structures. From numerical results, the role of the incident Mach number, the generation of spherical double Mach reflection, the maximum pressure from focusing, and discrepancies between axisymmetric and planar shock focusing are discussed in detail.

\section{Problem description}

The sketch of the toroidal shock wave focusing and computational domain are shown in Fig. 1, with the outer radius $D=0.5 \mathrm{~m}$ and the inner radius $d=0.4 \mathrm{~m}$. The toroidal shock wave is generated, at first, from the annual shock tube, and will diffract over the backward step after discharging and then generate the diffracting shock wave travelling toward the axis of symmetry. Finally, the diffracting shock waves will focus on the axis of symmetry and generate high 


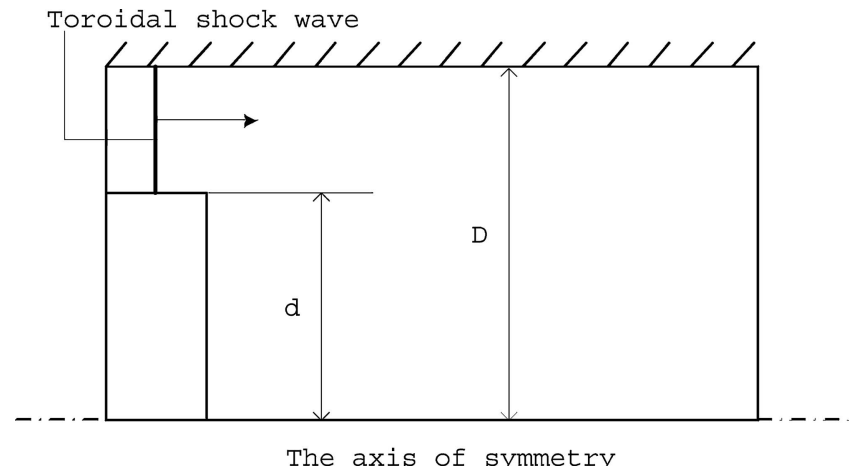

Fig. 1 The sketch of the toroidal shock wave focusing and computational domain with $D=0.5 \mathrm{~m}$ and $d=0.4 \mathrm{~m}$

temperature and pressure in a small region. The flow state is usually transient, but the flow properties have extraordinary peak values and can be applied in medical and industrial processes. Actually, the pressure and temperature on the focal point appear as singular points in mathematics, and can reach infinity neglecting viscosity. But taking into account viscosity and dissipation pressure and temperature in the focusing points are finite. In numerical simulations, the grid size may affect flow parameters at the focal point more or less, but the flow structures induced by focusing will remain the same.

\section{Governing equations and numerical methods}

In the numerical simulation, two-dimensional axisymmetric Navier-Stokes equations for a perfect gas are solved, which can be written as

$\frac{\partial U}{\partial t}+\frac{\partial F}{\partial x}+\frac{\partial G}{\partial r}+\frac{1}{r} S=\frac{\partial F_{\mathrm{v}}}{\partial x}+\frac{\partial G_{\mathrm{v}}}{\partial r}+\frac{1}{r} S_{\mathrm{v}}$,

where $U, E, F$, and $S$ denote the state variables, convection fluxes in each direction and sources, respectively, given by

$U=\left(\begin{array}{c}\rho \\ \rho u \\ \rho v \\ \rho e\end{array}\right), \quad F=\left(\begin{array}{c}\rho u \\ \rho u^{2}+p \\ \rho u v \\ (\rho e+p) u\end{array}\right)$,

$G=\left(\begin{array}{c}\rho v \\ \rho u v \\ \rho v^{2}+p \\ (\rho e+p) v\end{array}\right), \quad S=\left(\begin{array}{c}\rho v \\ \rho u v \\ \rho v^{2} \\ (\rho e+p) v\end{array}\right)$.

where $E_{\mathrm{v}}, F_{\mathrm{v}}$, and $S_{\mathrm{v}}$ denote the viscous flux and viscous source corresponding to $E, F$, and $S$, respectively. Primitive variables in the unknown $U$ are density $\rho$, velocity components $u$ and $v$, and total energy per unit volume $e$. Fluid pressure is $p$ and the equation of state for the perfect gas is given by

$e=\frac{p}{\gamma-1}+\frac{1}{2} \rho\left(u^{2}+v^{2}\right)$,

where $\gamma$, the specific heat ratio, is taken as 1.4.
The dispersion controlled dissipation scheme and the central difference are respectively applied to discretize the convection terms and the viscous terms. The DCD scheme has been demonstrated to be capable of predicting complex flow fields, such as shock wave interactions, without nonphysical oscillations and any need for artificial viscosity. The finite difference equations of the axisymmetric governing Eq. (1) discretized using the dispersion controlled dissipation scheme are given in semi-discrete form as follows:

$$
\begin{aligned}
\left(\frac{\partial U}{\partial t}\right)_{i, j}^{n}= & -\frac{1}{\Delta x}\left(H_{i+\frac{1}{2}, j}^{n}-H_{i-\frac{1}{2}, j}^{n}\right) \\
& -\frac{1}{\Delta r}\left(P_{i, j+\frac{1}{2}}^{n}-P_{i, j-\frac{1}{2}}^{n}\right)-S_{i, j}^{n},
\end{aligned}
$$

where

$$
\begin{aligned}
& H_{i+\frac{1}{2}, j}^{n}=F_{i+\frac{1}{2} L, j}^{+}+F_{i+\frac{1}{2} R, j}^{-}, \\
& P_{i, j+\frac{1}{2}}^{n}=G_{i, j+\frac{1}{2} L}^{+}+G_{i, j+\frac{1}{2} R}^{-},
\end{aligned}
$$

with

$$
\begin{aligned}
& F_{i+\frac{1}{2} L, j}^{+}=F_{i, j}^{+}+\frac{1}{2} \Phi_{\mathrm{A}}^{+} \operatorname{minmod}\left(\Delta F_{i-\frac{1}{2}, j}^{+}, \Delta F_{i+\frac{1}{2}, j}^{+}\right), \\
& F_{i+\frac{1}{2} R, j}^{-}=F_{i+1, j}^{-}-\frac{1}{2} \Phi_{\mathrm{A}}^{-} \operatorname{minmod}\left(\triangle F_{i+\frac{1}{2}, j}^{-}, \Delta F_{i+\frac{3}{2}, j}^{-}\right),
\end{aligned}
$$$$
\begin{aligned}
& G_{i, j+\frac{1}{2} L}^{+}=G_{i, j}^{+}+\frac{1}{2} \Phi_{\mathrm{B}}^{+} \operatorname{minmod}\left(\triangle G_{i, j-\frac{1}{2}}^{+}, \Delta G_{i, j+\frac{1}{2}}^{+}\right), \\
& G_{i, j+\frac{1}{2} R}^{-}=G_{i, j+1}^{-}-\frac{1}{2} \Phi_{\mathrm{B}}^{-} \operatorname{minmod}\left(\triangle G_{i, j+\frac{1}{2}}^{-}, \Delta G_{i, j+\frac{3}{2}}^{-}\right),
\end{aligned}
$$$$
\triangle F_{i+\frac{1}{2}, j}^{ \pm}=F_{i+1, j}^{ \pm}-F_{i, j}^{ \pm},
$$$$
\triangle G_{i, j+\frac{1}{2}}^{ \pm}=G_{i, j+1}^{ \pm}-G_{i, j}^{ \pm} \text {, }
$$

$F^{ \pm}=A^{ \pm} U$

$G^{ \pm}=B^{ \pm} U$,

$\Phi_{\mathrm{A}}^{ \pm}=I \mp \beta \Lambda_{\mathrm{A}}^{ \pm}$,

$\Phi_{\mathrm{B}}^{ \pm}=I \mp \beta \Lambda_{\mathrm{B}}^{ \pm}$,

In the equations presented above, the (.) $)^{+}$or $(.)^{-}$superscript signs denote vector flux splitting according to the StegerWarming method [7]. $I$ is a unit vector, $\beta=\Delta t / \Delta x$, and $\Lambda_{\mathrm{A}}$ and $\Lambda_{\mathrm{B}}$ are vectors that consist of eigenvalues of matrix $\mathrm{A}$ and $\mathrm{B}$, respectively. The time-marching integration was performed using a Runge-Kutta integration method of second-order accuracy. As the initial condition, the toroidal shock wave is travelling in the annular shock tube and the gas in front of the shock wave surface is at rest. As boundary conditions, no-slip and adiabatic conditions are used on the solid wall and free stream inlet and outlet flow are used on the two ports of the shock tube. 


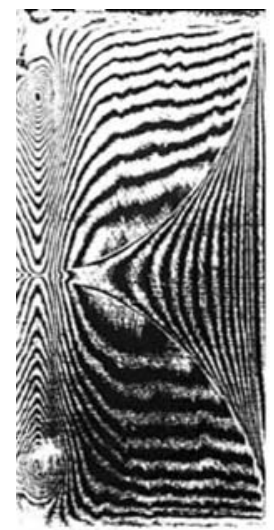

a

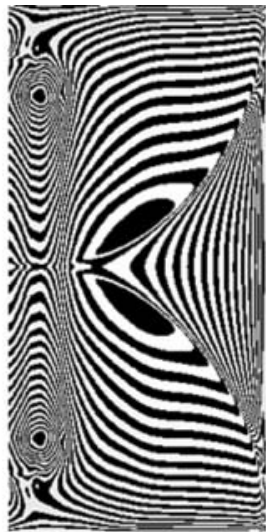

b

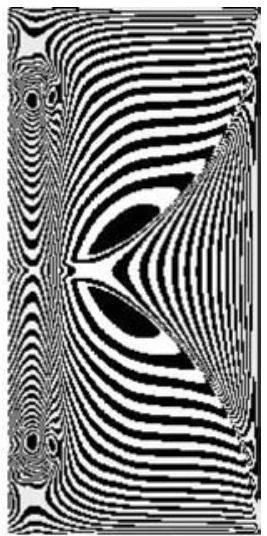

$\mathrm{C}$
Fig. 2 Interferograms of toroidal shock wave focusing for an incident Mach number of $M_{\mathrm{s}}=1.5$. a Experimental result; $\mathbf{b}$ numerical data with viscosity; $\mathbf{c}$ numerical data without viscosity

\section{Solution verification}

To validate the numerical method used in the present simulation, the toroidal shock wave focusing for an incident Mach number of $M_{\mathrm{s}}=1.5$ was computed. Numerical interferograms were constructed from the numerical data by integrating the density of the flow field [8] and are compared directly with the experiment [5], as shown in Fig. 2, where the experimental result is in the left part, the numerical data with viscosity in the middle and that without viscosity in the right. The figures show the wave structure when the imploding shock wave is up to focus on the axis of symmetry. From the figures, it can be observed that not only the number of fringes but also the distribution of individual fringes are identical, so it can be concluded that the code is credible and can be used to investigate the shock wave focusing. Near the solid wall the fringe number in the inviscid case shown in Fig. $2 \mathrm{c}$ is larger than that of the experimental result, and taking into account viscosity, the departure will be improved greatly, see Fig. $2 b$.

\section{Numerical results and discussion}

In the following discussion, several issues will be emphasized and analyzed in detail. The first one is the process of the toroidal shock wave focusing, from which wave structures and spacial flow states are observable. The second is the role of the incident Mach number in shock focusing and it is a key parameter for diametrical changes in shocked flows. The third is the converging process of diffracting shock waves, in which the Mach number of the diffracting shock waves is increased. It is demonstrated that the process plays a key role in shock focusing. The last one is in the high Mach number case in which the shock focusing will generate two effective focal points and a strong supersonic jet that impacts on the Mach stem and renders it convex.

\subsection{Toroidal shock wave focusing}

The second case, for a Mach number of $M_{\mathrm{S}}=4.0$, is simulated and the process of the toroidal shock wave focusing is plotted in Fig. 3. In this figure, density contours are shown in the upper half and the pressure contours in the lower half. The toroidal shock wave diffracts over the backward step after discharging from the annular shock tube to the cylindrical chamber and implodes toward the axis of symmetry as shown in Fig. 3a. It can be observed that the wave front of the diffracting shock wave is an arc and there is a short Mach stem close to the backward step. Meanwhile, a secondary shock wave develops behind the diffracting shock wave because of the locally developed supersonic flow. The part of the wave front reaching the axis of symmetry first is not the Mach stem, because the wave front is curved much more toward. After focusing, the shock wave reflects from the axis of symmetry, as shown in Fig. 3b. The pattern of the reflected shock wave is, at first, regular reflection, but with the lapse of time it quickly changes to Mach reflection and a short Mach stem occurs. Meanwhile, the shock wave reflected from the axis of symmetry, like a cylindric blast wave generating from the high pressure induced by focusing, propagates to the outside. From Fig. 3b to 3c, the Mach stem is getting longer and longer, and the part near the axis is curved forward and becomes convex. It is believed that the blast wave behind drives out the special Mach stem. The wave structure, called spherical double Mach reflection in some papers, appears to be self-similar in Fig. $3 \mathrm{c}$ and $3 \mathrm{~d}$ and behind the convex Mach stem there are very complex interactions among shock waves, slip lines, and eddies.

\subsection{The role of the incident Mach number}

To investigate the role of the incident Mach number, four cases are computed by varying the incident Mach number. Isobars and isopycnics of the numerical results of shock wave focusing for the incident Mach numbers of $M_{\mathrm{s}}=2.0$, 3.0, 4.0, and 5.0 are plotted in Fig. 4, respectively. From the figure it is observable that there are great differences in the wave structure. For the case of Mach number 2.0, the single Mach reflection develops and as the incident shock propagates, the Mach stem becomes longer and longer, and appears nearly planar, as shown in Fig. 4a. When increasing the incident Mach number, the Mach stem becomes convex gradually and the spherical double Mach reflection appears more and more obvious to be seen from Fig. 4b to $4 \mathrm{~d}$. A new upstream shock wave, being vertical to the axis and like a shock disk, develops behind the Mach stem, and its edge sucks in the low pressure core of the vortex ring, as shown in Fig. $4 \mathrm{c}$ and d. It is concluded that the Mach number of the incident toroidal shock wave plays a vital role in the wave structure development of focusing.

It seems that the spherical double Mach reflection is a distinctive phenomenon in focusing. The question is what is the reason for the convex Mach stem on generation. From the numerical results it is found that behind the curved Mach 

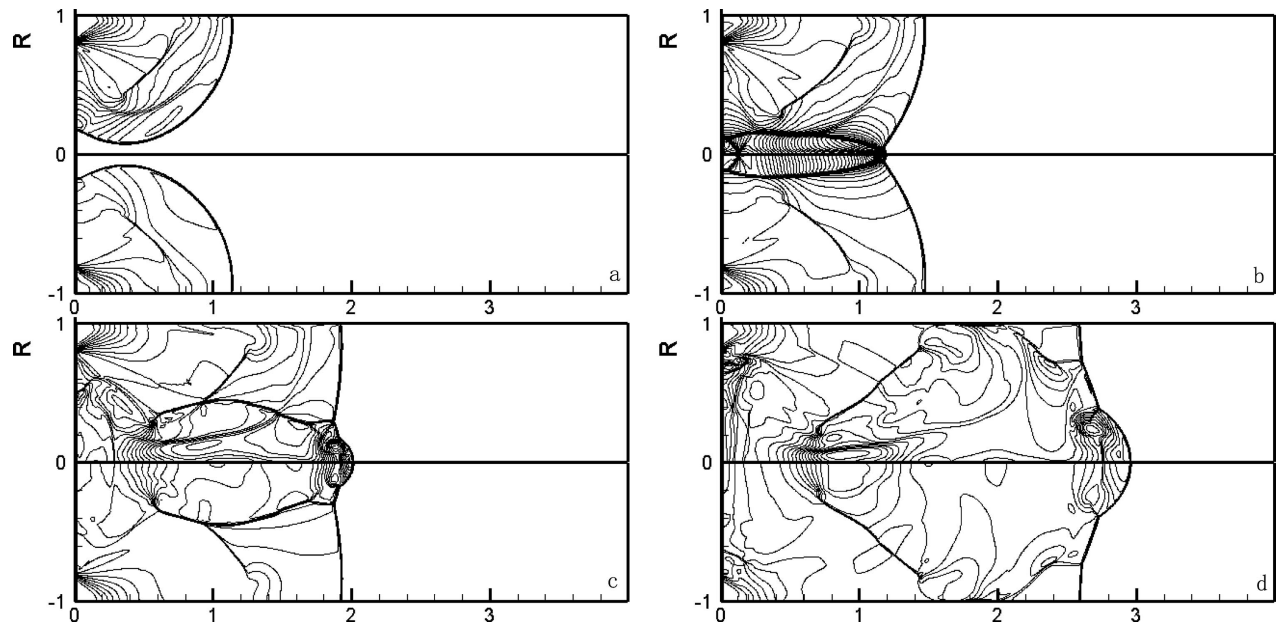

Fig. 3 Isobars (lower half) and isopycnics (upper half) of toroidal shock wave focusing for $M_{\mathrm{s}}=4.0$ : a $59 \mu \mathrm{s}$; b $82 \mu \mathrm{s}$; c $114 \mu \mathrm{s} ; \mathbf{d} 158 \mu \mathrm{s}$
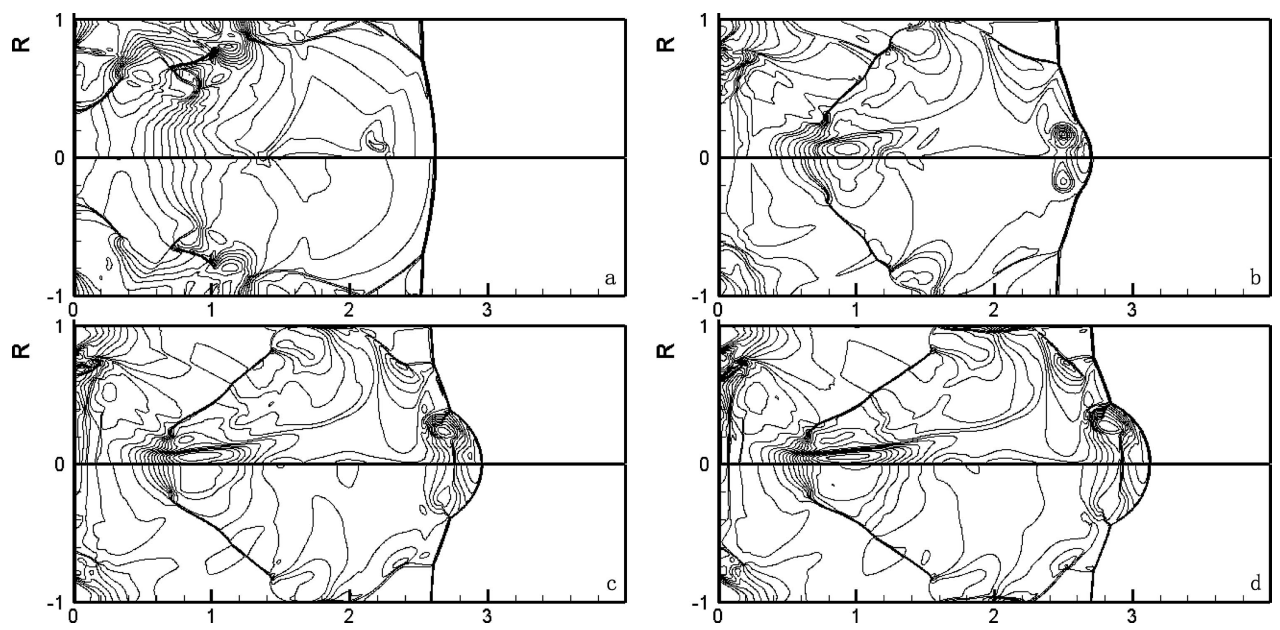

Fig. 4 Isobars (lower half) and isopycnics (upper half) of shock wave focusing for various incident Mach numbers: $\mathbf{a} M_{\mathrm{S}}=2.0,219 \mu \mathrm{s}$; $\mathbf{b}$ $M_{\mathrm{S}}=3.0,181 \mu \mathrm{s} ; \mathbf{c} M_{\mathrm{S}}=4.0,158 \mu \mathrm{s}$, and $\mathbf{d} M_{\mathrm{S}}=5.0,121 \mu \mathrm{s}$

stem, a region of great particle velocity exists along the axis of symmetry. To show this, the velocity in $x$-direction on the axis of symmetry and the line of $x=1.9$ are shown respectively in Fig. 5 when the spherical double Mach reflection just occurs as shown in Fig. 3c. Figure 5 shows that the peak velocity appears at the position of $r=0.0$ and $x=1.9$, where the local Mach number can reach values as high as 11.3. Along the line of $x=1.9$, the velocity in $x$-axis direction increases linearly and reaches its maximum value at the wave front. So, it can be concluded that shock focusing induces a jet around the axis of symmetry, and the jet then impacts on the Mach stem and renders it curved. Finally, the spherical double Mach reflection develops. To support this explanation, the distribution of the velocity in $x$-direction along a vertical line behind the Mach stem is shown in Fig. 6 when the Mach reflection just occurs as shown in Fig. 3b, and a similar velocity profile for the low incident Mach number of 1.5 is also displayed in the same figure. Obviously, there is a great velocity gradient near the axis in the high Mach number case, which implies a strong supersonic jet exists here. The jet impacts on the Mach stem ahead and renders it convex. The interaction generates an inflection point, which is the second triple point for the wave system.

The time history of the maximum pressure along the axis of symmetry for incident Mach numbers of (a) 2.0, (b) 3.0, (c) 4.0, and (d) 5.0 is recorded and shown in Fig. 7. The figure shows that as the incident Mach number increases, the peak pressure on the axis increases rapidly. For example, if the Mach number of the toroidal shock wave changes from 2.0 to 5.0 , the post-shock pressure behind the wave front increases by 6.4 times, but the peak pressure increases by 15.5 times. Therefore, the toroidal shock wave focusing is an effective way to get high pressure and temperature.

In Fig. 7, some unusual phenomena are worth to be pointed out. There are two pressure extrema on the axis of symmetry for high Mach numbers. The two pressure extrema indicate two effective focal pressures: The first one is 

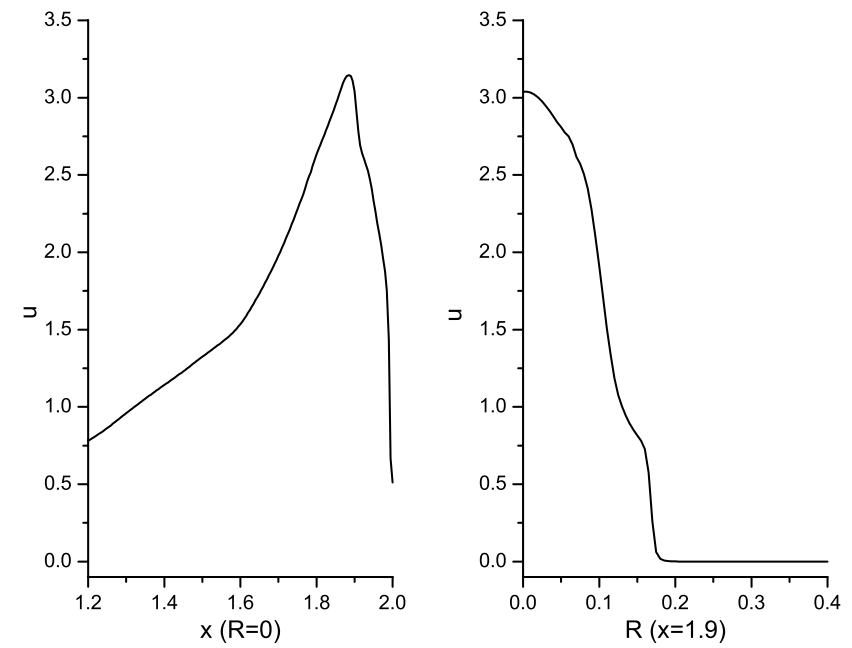

Fig. 5 The velocity distribution along the axis and the line $x=1.9$ for $M_{\mathrm{S}}=4.0$

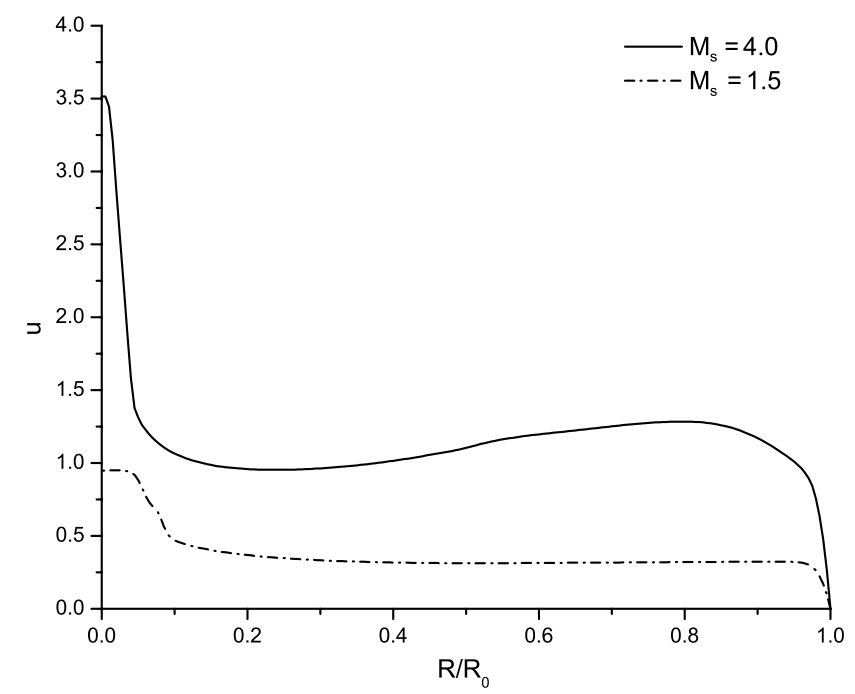

Fig. 6 The velocity distribution in the $x$-direction just behind of shock wave front for $M_{\mathrm{S}}=1.5$ and 4.0

located near the wall at the axis of symmetry and the second is a certain distance away from the wall, in front of where the diffracting shock wave arrives first. Theoretically speaking, the pressure on the axis depends on two factors: the incident shock angle and the incident Mach number. Near the wall, the incident Mach number is zero and the incident Mach number is higher than the adjacent shock because of the Mach stem induced by overexpansion. Furthermore, before this focal point, the velocity is in negative $x$-direction and then stagnated here by the wall. So near the wall the first focal point is generated. The second focal point is located in front of the first arriving point, from where the incident shock angle increases gradually from zero and the incident Mach number also ascends along the $x$-direction. In the beginning the higher Mach number can offset the bigger incident angle excessively and generates a higher pressure.

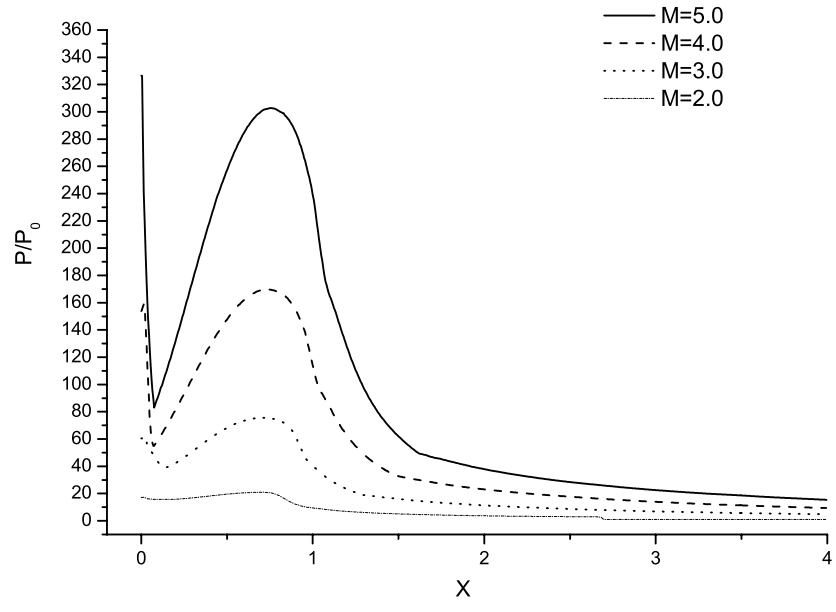

Fig. 7 The time history of the maximum pressure profile for incident Mach numbers of $M_{\mathrm{s}}=2.0,3.0,4.0$, and 5.0

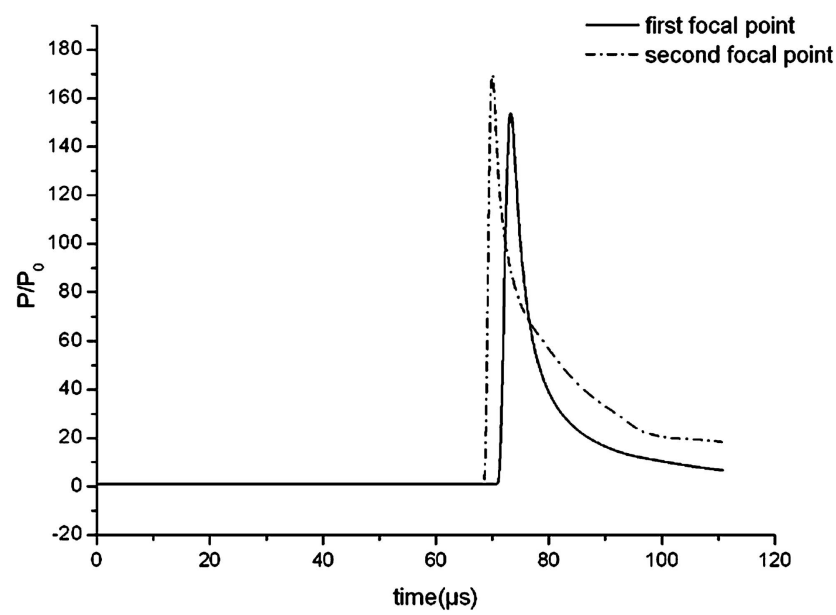

Fig. 8 The pressure on the two effective focusing points for $M_{\mathrm{s}}=4.0$

But because the angle increases more and more rapidly, at a certain point the effect of both the factors is opposite completely and the pressure reaches its peak. So the peak point is the second effective point that is located a little in front of the first arriving point.

The pressure on the two effective focal points is shown as function of time in Fig. 8. Both the pressures reach their peak value when the shock wave arrives, and then decreases rapidly. The pressure pulse lasts only a few microsecond but can reach a level being hundred times higher than the pressure in front of shock wave front. It is also noted that if the Mach number is less than 4.0, the second focusing pressure is higher than the first. When the incident Mach number increases to 5.0, the bigger focusing pressure appears at the first focusing point, as shown in Fig. 7. So, there must be a threshold Mach number between 4.0 and 5.0, at which the position of the bigger focusing pressure alters. In conclusion, increasing the incident Mach number affects not only the focusing pressure, but also the focal points that may move from an uncertain point to a fixed point. 


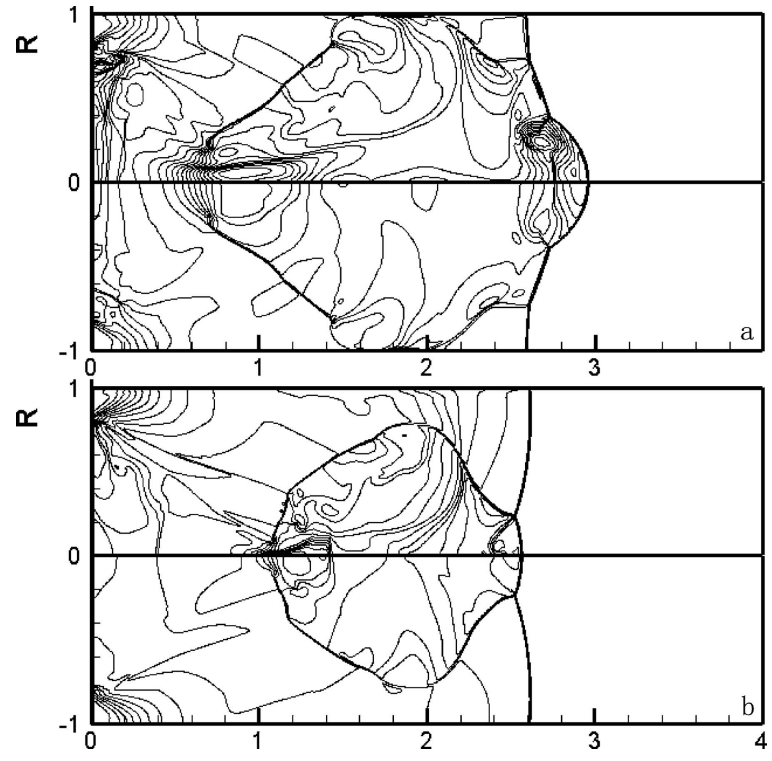

Fig. 9 Isobars (lower half) and isopycnics (upper half) after focusing for $M_{\mathrm{s}}=4.0$ : a axisymmetric flow, $158 \mu \mathrm{s}$; b planar flow, $169 \mu \mathrm{s}$

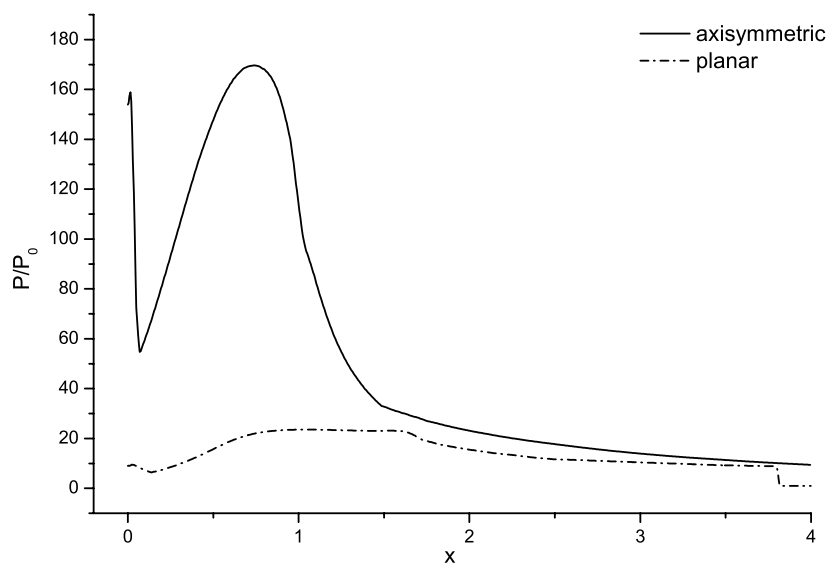

Fig. 10 The historic maximum pressure for $M_{\mathrm{S}}=4.0$ in the axisymmetric and planar flow

\subsection{The axisymmetric shock focusing}

To explore the mechanism of the axisymmetric shock focusing, a two-dimensional planar shock focusing is also simulated. During the imploding process before the focusing the flow structure is almost the same as the axisymmetric case. But after focusing, the flow structure is quite different, as shown in Fig. 9. Obviously for the same incident Mach number 4.0, there is no spherical double Mach reflection in the planar flow. So it can be deduced that the maximum pressure on the plane of symmetry must be lower to generate the supersonic jet, and a comparison of the historic maximum pressures for two cases is demonstrated in Fig. 10. The figure shows that the two peak pressure values in the planar case are both much lower than the corresponding focal

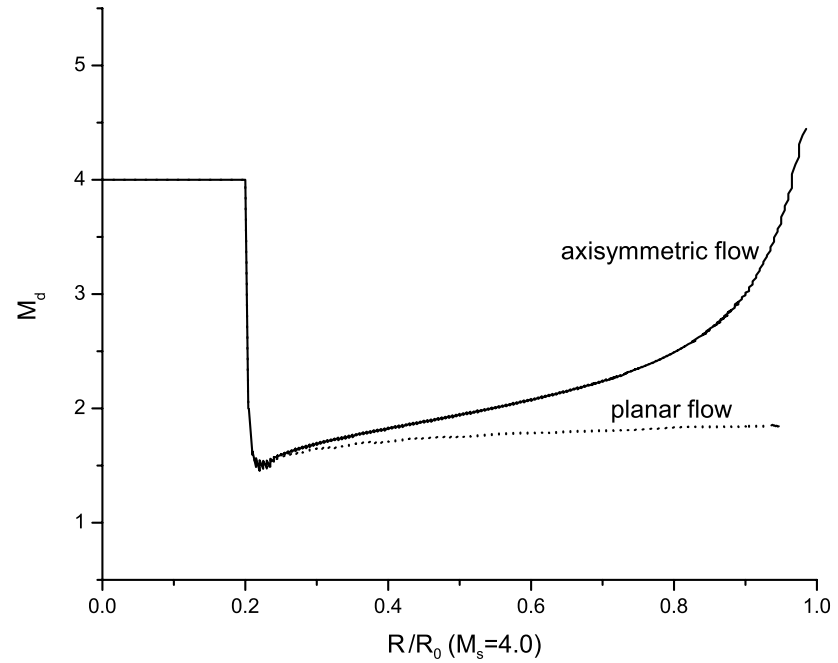

Fig. 11 Mach number of diffracting shock wave for $M_{\mathrm{S}}=4.0$ when converging inside

values in the axisymmetric case. The difference between the two cases must be generated before shock focusing. So the diffracting shock wave Mach number near the backward step during the diffracting shock wave implodes toward the axis is recorded, as shown in Fig. 11. Before toroidal shock waves diffract, the Mach number is the incident Mach number 4.0. Once the diffracting process starts, the Mach number drops down to about 1.5 in both cases. During the implosion, the diffracting shock wave in the two cases behaves differently. In the axisymmetric case, the Mach number increases more and more rapidly and finally exceeds the incident one. However, in the planar case, the Mach number is almost constant before focusing, being equal to the value at the beginning of the diffraction. This indicates that the accelerating process in the axisymmetric case can overcompensate the decreased Mach number induced by the diffraction, while in the planar case there is no accelerating process and the diffracting shock wave Mach number keeps close to 1.5. In other words, the diffracting and accelerating processes influence the focusing oppositely. The diffracting produces the diffracting shock wave that implodes, but reduces the Mach number perishingly. The accelerating occurs in the axisymmetric case when the diffracting shock wave implodes, which is the key factor to generate effective shock focusing.

\section{Conclusions}

From the discussion above, the numerical investigation can be summarized as follows: The toroidal shock wave focusing is an effective way to get high pressure and temperature pulses being in a microsecond magnitude. The converging process of diffracting shock waves, which increases the Mach number of the diffracting shock waves, plays a key role in shock focusing. In the high Mach number case the 
shock focusing will generate two effective focal points and a strong supersonic jet that impacts on the Mach stem and renders it convex. The process induces a spherical double Mach reflection. The very complicated interactions among shock waves, contact surfaces and eddies are observable in the spherical double Mach reflection that needs more future work to investigate.

Acknowledgements This work has been supported by the National Natural Science Foundation of China through Grant No. 90205027. The authors thank the editor and anonymous referees for their valuable comments and help in presenting this work.

\section{References}

1. Sod, G.A.: A numerical study of a converging cylindrical shock. J. Fluid Mech. 73, 651-671 (1977)
2. Takayama, K., Kieine, H., Grönig, H.: An experimental investigation of the stability of converging cylindrical shock waves in air. Exp. Fluid 5, 315-322 (1987)

3. Grönig, H.: Past, present and future of shock wave focusing research. In: Proceeding of the Int. Workshop on Shock Wave Focusing, Sendai, Japan, pp. 1-37 (1989)

4. Jiang, Z., Takayama, K.: Reflection and focusing of toroidal shock waves from coaxial annular shock tubes. Comput. Fluids 27, 553562 (1998)

5. Hosseini, H.S.R., Takayama, K.: Study of shock wave focusing and reflection over symmetrical axis of a compact vertical co-axial diaphragmless shock tube. In: Proceedings of 23rd ISSW, Vol. 2, pp. 1550-1557 (2001)

6. Jiang, Z.: On dispersion-controlled principles for non-oscillatory shock-capturing schemes. Acta Mechan. Sin. 20, 1-15 (2004)

7. Steger, J., Warming, R.: Flux vector splitting of the inviscid gasdynamic equations with applications to finite difference methods. J. Comput. Phys. 40, 263-293 (1981)

8. Jiang, Z., Takayama, K.: An investigation into the validation of numerical solution of complex flowfields. J. Comput. Phys. 151, 479-497 (1999) 